\title{
Calculation of interfacial tension from density of states
}

\author{
Tushar S. Jain and Juan J. de Pablo ${ }^{\text {a) }}$ \\ University of Wisconsin-Madison, Department of Chemical Engineering, Madison, Wisconsin 53706
}

(Received 19 September 2002; accepted 4 December 2002)

\begin{abstract}
A density of states Monte Carlo formalism is used to calculate the interfacial tension of a simple fluid (3D Ising model) and of free-standing polymer films on a lattice. Good agreement is found between the results of these calculations and literature values. The proposed approach is efficient and provides the surface tension over a wide range of temperature from a single simulation. It has the additional advantage of being equally applicable to systems interacting through continuous potential energy functions, discontinuous functions, and systems on a lattice. (C) 2003 American Institute of Physics. [DOI: 10.1063/1.1540613]
\end{abstract}

\section{INTRODUCTION}

The interfacial properties of a system are of considerable interest, both from a technological and a fundamental perspective. As the scale of devices shrink to the nanometer length scale, interfacial properties are expected to play an important role in their behavior. A number of recent studies suggest that the properties of thin polymer films exhibit fundamentally different properties from the bulk on account of the interfaces present in these systems (e.g., different glass transition temperature ${ }^{1,2}$ ). In order to study and understand the properties of ultra small systems, it is important to develop or refine our ability to calculate the interfacial tension of relevant interfaces.

One of the most common methods used to calculate interfacial tension is based on the normal and tangential pressure profiles of an interfacial system. ${ }^{3-5}$ For continuous and differentiable potentials, these pressure profiles can be calculated by using the Kirkwood or Harashima conventions. ${ }^{6-8}$ However, in the absence of continuously differentiable potentials, such conventions cannot be used. For some systems, an analysis of the capillary wave spectrum ${ }^{9,10}$ has been proposed to extract the surface tension. Calculations based on capillary wave theory, however, require large system sizes for the wave spectrum to get established and relax, as well as simulations of different system sizes to study the scaling of interfacial width. In the case of polymeric systems at temperatures far from the critical point or in the vicinity of the glass transition temperature, the relevant relaxation times become extremely long, thereby requiring long simulations. A different method used to obtain the interfacial tension is based on multicanonical simulations ${ }^{11}$ in the grand-canonical ensemble. ${ }^{12}$ Such a method, however, is limited to situations where grand-canonical simulations are feasible. For long polymers, the required insertion and deletion of chain molecules can be problematic, and alternative approaches could be of use.

In this paper, we propose a procedure for calculation of interfacial tensions that relies on a density of states algorithm and the difference in free-energy between bulk and interfa-

a) Author to whom correspondence should be addressed. cial systems. We find that this method is computationally efficient, accurate, and permits study of both continuous and discontinuous potentials, as well as systems on a lattice.

\section{METHODS}

\section{A. Calculating the interfacial tension}

For an interfacial system, the Helmholtz free-energy of the system can be written as

$$
F=-p V+\mu N+\gamma A,
$$

where $F$ is the Helmholtz free-energy, $p$ is the pressure, $V$ is the volume, $\mu$ is the chemical potential, $N$ is the number of molecules, $\gamma$ is the interfacial tension, and $A$ is the interfacial area of the system. This equation can be rearranged as follows:

$$
\gamma=\frac{F+p V-\mu N}{A} .
$$

The interfacial tension of a system at temperature $T$ can be determined from the knowledge of the coexistence properties $[\mu(T), p(T)]$ and the free-energy of the system $F(T)$. In this work, a density of states formalism is used to calculate $F$ for an interfacial system and to determine its coexistence properties.

In the case of a grand-canonical simulation at coexistence, the probability distribution of the density, $P(\phi)$, exhibits two peaks corresponding to the coexisting phases. Configurations corresponding to intermediate densities typically involve the formation of an interface and, therefore, have a much lower probability of occurring (due to the associated free-energy cost of the interface formation). The free-energy, $F(\phi)$, associated with this probability distribution can be written as ${ }^{12}$

$$
F(\phi)=-k_{B} T \log P(\phi) .
$$

The difference in free-energy between that of the coexisting phases, $F_{\text {coex }}$, and that of the intermediate configurations involving the interface, $F_{\text {int }}$, provides another route for calculation of the interfacial tension. Formally, this can be expressed as 


$$
\gamma=\frac{F_{\text {int }}-F_{\text {coex }}}{A} .
$$

\section{B. Density of states}

In order to calculate $F(T)$ for an interfacial system we use a density of states algorithm. The details of the algorithm can be found elsewhere. ${ }^{13,14}$ In brief, a random walk is performed in energy space with probability proportional to $1 / \Omega(U)$, where $U$ is the potential energy and $\Omega(U)$ is the density of states of the system. The simulation initially starts with $\Omega(U)=1$ for all $U$, and gradually converges to the required $\Omega(U)$ using a set procedure. Once $\Omega(U)$ is obtained (up to the required accuracy), the thermodynamic properties can be calculated from the partition function. The Helmholtz free-energy can be calculated as

$$
F=-k_{B} T \ln \left[\sum_{U} \Omega(U) \exp (-\beta U)\right] .
$$

This formalism has been extended to the grand canonical ensemble, ${ }^{15}$ where the number of particles in the system is allowed to fluctuate. In this case, the two-dimensional density of states $\Omega(N, U)$ is calculated, where $N$ is the number of particles in the system. The probability of observing a system having $N$ particles is given by

$$
P(N)=\frac{\Sigma_{U} \Omega(N, U) \exp (-\beta U+\beta \mu N)}{\Sigma_{N} \Sigma_{U} \Omega(N, U) \exp (-\beta U+\beta \mu N)} .
$$

At coexistence, this probability distribution exhibits two peaks of equal area. The chemical potential $\mu$ at coexistence can be determined by manipulating the probability distribution function (through $\mu$ ) until the equal-area criterion is met. The coexistence pressure $p$ can then be calculated from $^{15}$

$$
\left\langle p_{V}\right\rangle=\frac{k_{B} T}{V} \ln \sum_{N \leqslant N_{o}} \sum_{U} \Omega(N, U) \exp (-\beta U+\beta \mu N)
$$

and

$$
\left\langle p_{L}\right\rangle=\frac{k_{B} T}{V} \ln \sum_{N>N_{o}} \sum_{U} \Omega(N, U) \exp (-\beta U+\beta \mu N),
$$

where $N_{o}$ is a threshold number of particles such that for $N \leqslant N_{o}$ the system is considered to be a vapor, and for $N$ $>N_{o}$ the system is considered to be a liquid.

The algorithm employed here to calculate the density of states gives the relative density of states. In the case of a grand-canonical simulation, the exact value of the density of states is known to be unity for a fully-occupied system, thereby providing an absolute value for the density of states. However, for the interfacial system, only relative freeenergies are obtained ( $F$ is only known to within an additive constant).

\section{Model}

We have studied two systems on a simple cubic lattice of unit length $\sigma$. The occupied sites on the lattice interact via a nearest-neighbor potential energy of magnitude $-\epsilon$.

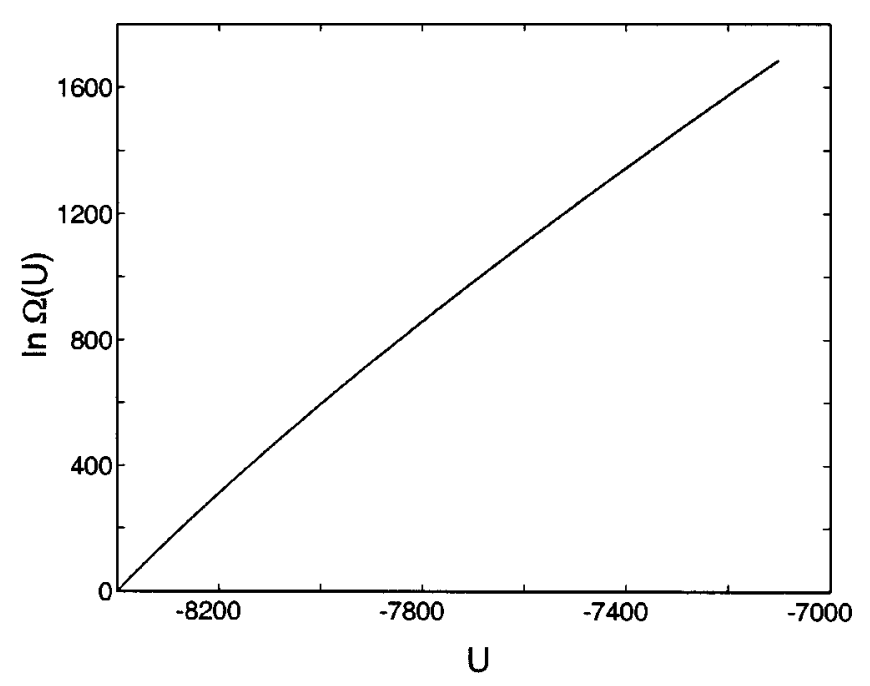

FIG. 1. Canonical density of states for a simple fluid (3D Ising model) thin film.

In the first system molecules occupy an individual lattice site. (This model is equivalent to the 3D Ising model.) The canonical density of states simulation was performed for a system of size $30 \times 15 \times 15$ comprising 3000 occupied lattice sites. The grand-canonical density of states simulation was performed on a smaller system of size $8 \times 8 \times 8$.

The second system consists of free-standing polymer films on a cubic lattice. The polymer chains are represented by self-avoiding random walks. The details of this system and the methods used for equilibration can be found elsewhere. ${ }^{16}$ The chain length $L$ considered here is 100 sites. The number of chains $N_{c}$ used in this work is 125. All the results are reported in reduced variables, i.e., temperature $T^{*}=k T / \epsilon$ and density $\phi=N \sigma^{3} / V$.

\section{RESULTS}

Figure 1 shows the density of states obtained for a film of the simple fluid. Figure 2 shows the two-dimensional density of states used for the calculation of phase coexistence properties. Using Eq. (3), we have plotted $F(\phi)$ for $T^{*}$ $=0.65$ in Fig. 3. Note that the free energy in the interfacial region is flat, thereby indicating that the system size employed in this example is sufficiently large. The values of $\gamma$ obtained are in good agreement with literature results and demonstrate the correctness of the method proposed here. In Fig. 4, the values of $\gamma$ obtained from Eqs. (2) and (4) are compared to those reported in the literature. ${ }^{17-19}$ The latter results were obtained from a virtual site removal strategy ${ }^{17}$ and by thermodynamic integration, ${ }^{18,19}$ respectively. Results from thermodynamic integration are expected to be more accurate due to limitations of the site removal strategy. ${ }^{17}$ The agreement between our calculations and the values reported in literature is excellent over the entire temperature range considered in this study.

The method proposed here provides a means of calculating $\gamma$ as a continuous function of temperature from a single simulation. One should note, however, that the free-energy obtained from the density of states of the interfacial system is accurate to within an additive constant. This method there- 


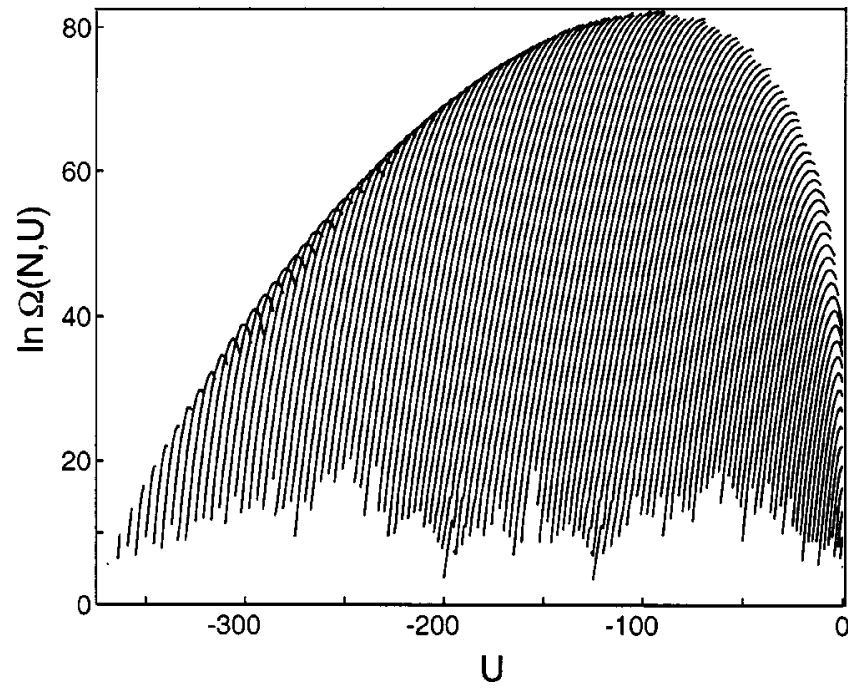

FIG. 2. Grand canonical density of states for a lattice fluid (3D Ising model). Each individual line represents the density of states at a constant value of $N$. For clarity, the density of states shown in this figure correspond to a lattice of size $5 \times 5 \times 5$. The results shown in Figs. 3 and 4 were obtained on a lattice of size $8 \times 8 \times 8$.

fore requires calibration with one point to arrive at absolute values of $\gamma$. We have used the value of $\gamma$ at $T^{*}=0.75$ (from the literature ${ }^{18}$ ) to calibrate our data. In the case of the grandcanonical density-of-states simulations, the absolute values of $\Omega(N, U)$ are known and the interfacial tension obtained from Eq. (4) does not require a calibration point.

For polymer chains we have determined the coexistence chemical potential through the incremental chain insertion method. ${ }^{20}$ Since the temperatures studied in this work are far from the critical point, we have assumed the value of the coexistence pressure to be $p=0$. We have considered three different film thicknesses to verify the validity of our results; the value of $\gamma$ should not be a function of film thickness. In the absence of a calibration point for this system, we have plotted the change in interfacial tension $\Delta \gamma$ as a function of $T^{*}$ for the three films (Fig. 5). We find that the curves for the

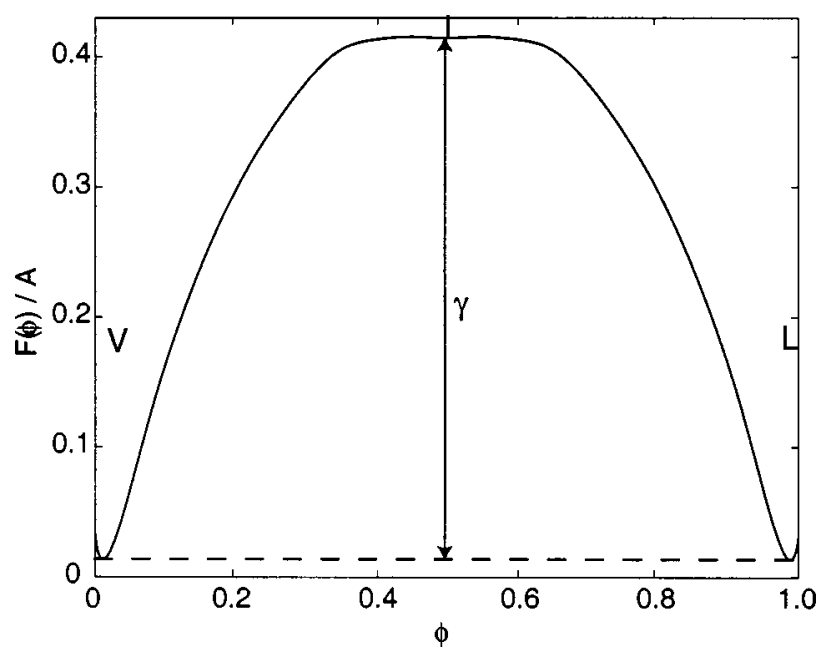

FIG. 3. Free-energy $F(\phi)$ from a grand-canonical ensemble simulation, showing the free-energy barrier due to interface formation. The symbols $L$, $V$, and $I$ refer to liquid, vapor, and interfacial configurations, respectively.

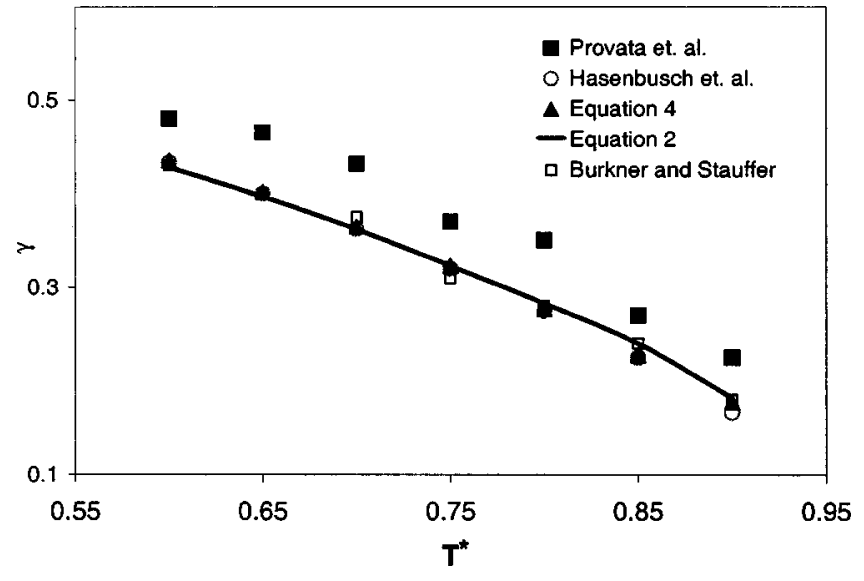

FIG. 4. Interfacial tension $\gamma$ for a simple lattice fluid (3D Ising model).

different thicknesses overlap with each other, indicating that the temperature dependence of $\gamma$ is indeed independent of thickness.

\section{CONCLUSIONS}

We have demonstrated the use of a density of states formalism to calculate the interfacial tension of monomers and polymers on a lattice. The agreement obtained between this method and published literature values is excellent. In the case of a simple lattice fluid, the grand canonical density of states can be obtained with modest computational efforts and provides two routes for calculation of $\gamma$ [through Eqs. (2) and (4)]. For long polymer chains, the calculation of the grand canonical density-of-states is more demanding and therefore only Eq. (2) was used in this work.

The computational cost and time involved in these methods is modest compared to that of existing methods for calculation of interfacial tension. This is especially true for polymeric systems, where large systems are required for cap-

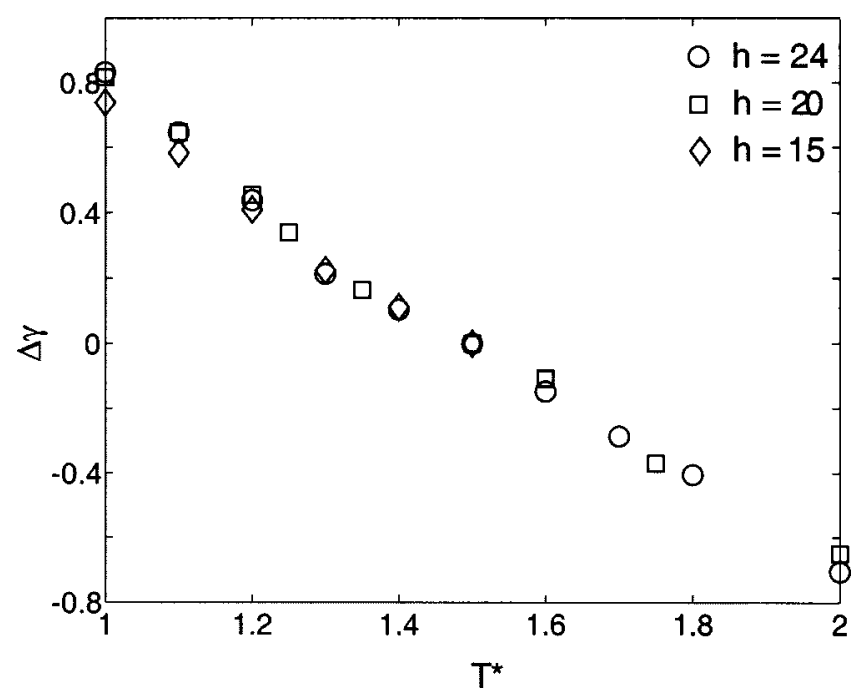

FIG. 5. Change in interfacial tension $\gamma$ with temperature for polymer chains of 100 segments on a cubic lattice. Results are shown for three different thicknesses $h$. 
illary waves to form and equilibrate, and where methods based on virtual site removal strategy have not been explored.

\section{ACKNOWLEDGMENTS}

This work is supported by the Division of Chemical Sciences, Office of Basic Energy Sciences, Office of Science, of the United States Department of Energy. Support from the Semiconductor Research Corporation is also acknowledged.

${ }^{1}$ D. S. Fryer et al., Macromolecules 34, 5627 (2001).

${ }^{2}$ E. Dalnoki-Veress et al., Phys. Rev. E 63, 031801 (2001).

${ }^{3}$ F. Varnik, J. Baschnagel, and K. Binder, J. Chem. Phys. 113, 4444 (2000).

${ }^{4}$ J. Alejandre, D. Tildesley, and G. Chapela, J. Chem. Phys. 102, 4574 (1995).

${ }^{5}$ J. L. Rivera, J. Alejandre, S. K. Nath, and J. J. de Pablo, Mol. Phys. 98, 40 (2000).
${ }^{6}$ B. Todd, D. Evans, and P. Daivis, Phys. Rev. E 52, 1627 (1995).

${ }^{7}$ J. Irving and J. Kirkwood, J. Chem. Phys. 18, 817 (1950).

${ }^{8}$ A. Harasima, Adv. Chem. Phys. 1, 203 (1958).

${ }^{9}$ K. Werner, F. Schmid, M. Müller, and K. Binder, Phys. Rev. E 59, 728 (1999).

${ }^{10}$ M. Müller and M. Schick, J. Chem. Phys. 105, 8885 (1996).

${ }^{11}$ B. Berg and T. Neuhaus, Phys. Rev. Lett. 68, 9 (1992).

${ }^{12}$ M. Müller and L. MacDowell, Macromolecules 33, 3902 (2000).

${ }^{13}$ T. S. Jain and J. J. de Pablo, J. Chem. Phys. 116, 7238 (2002).

${ }^{14}$ F. Wang and D. Landau, Phys. Rev. Lett. 86, 2050 (2001).

${ }^{15}$ Q. R. Yan, R. Faller, and J. J. de Pablo, J. Chem. Phys. 116, 8745 (2002).

${ }^{16}$ T. S. Jain and J. J. de Pablo, J. Chem. Phys. 35, 2167 (2002).

${ }^{17}$ A. Provata, D. Prassas, and D. N. Theodorou, J. Chem. Phys. 107, 5125 (1997).

${ }^{18}$ M. Hasenbusch and K. Pinn, Physica A 203, 189 (1994).

${ }^{19}$ E. Bürkner and D. Stauffer, Z. Phys. B: Condens. Matter 55, 241 (1983).

${ }^{20}$ S. Kumar, I. Szleifer, and A. Z. Panagiotopoulos, Phys. Rev. Lett. 66, 2935 (1991). 